\title{
Vortex patterns in a fast rotating Bose-Einstein condensate
}

\author{
Amandine Aftalion, Xavier Blanc \\ Laboratoire Jacques-Louis Lions, Université Paris 6, \\ 175 rue du Chevaleret, 75013 Paris, France. \\ Jean Dalibard \\ Laboratoire Kastler Brossel, 24 rue Lhomond, 75005 Paris, France
}

(Dated: July 23, 2018)

\begin{abstract}
For a fast rotating condensate in a harmonic trap, we investigate the structure of the vortex lattice using wave functions minimizing the Gross Pitaveskii energy in the Lowest Landau Level. We find that the minimizer of the energy in the rotating frame has a distorted vortex lattice for which we plot the typical distribution. We compute analytically the energy of an infinite regular lattice and of a class of distorted lattices. We find the optimal distortion and relate it to the decay of the wave function. Finally, we generalize our method to other trapping potentials.

PACS numbers: 03.75.Lm,05.30.Jp
\end{abstract}

The rotation of a macroscopic quantum fluid is a source of fascinating problems. By contrast with a classical fluid, for which the equilibrium velocity field corresponds to rigid body rotation, a quantum fluid described by a macroscopic wave function rotates through the nucleation of quantized vortices [1, 2]. A vortex is a singular point (in 2 dimensions) or line (in 3 dimensions) where the density vanishes. Along a contour encircling a vortex, the circulation of the velocity is quantized in units of $h / m$, where $m$ is the mass of a particle of the fluid.

Vortices are universal features which appear in many macroscopic quantum systems, such as superconductors or superfluid liquid helium. Recently, detailed investigations have been performed on rotating atomic gaseous Bose-Einstein condensates. These condensates are usually confined in a harmonic potential, with cylindrical symmetry around the rotation axis $z$. Two limiting regimes occur depending on the ratio of the rotation frequency $\Omega$ and the trap frequency $\omega$ in the $x y$ plane. When $\Omega$ is notably smaller than $\omega$, only one or a few vortices are present at equilibrium $[3,4]$. When $\Omega$ approaches $\omega$, since the centrifugal force nearly balances the trapping force, the radius of the rotating gas increases and tends to infinity, and the number of vortices in the condensate diverges [ [5, [6, 7, 8].

As pointed out by several authors, the fast rotation regime presents a strong analogy with Quantum Hall physics. Indeed the one-body hamiltonian written in the rotating frame is similar to that of a charged particle in a uniform magnetic field. Therefore the ground energy level is macroscopically degenerate, as the celebrated Landau levels obtained for the quantum motion of a charge in a magnetic field. There are two aspects in this connection with Quantum Hall Physics. Firstly, when the number of vortices inside the fluid remains small compared to the number $N$ of atoms, we expect that the ground state of the system will correspond to a BoseEinstein condensate, described by a macroscopic wave function $\psi(\boldsymbol{r})$. This situation has been referred to as 'mean field Quantum Hall regime' 9, 10, 11, 12, 13, 14].
Secondly, when $\Omega$ approaches $\omega$ even closer, the number of vortices reaches values comparable to the total number of atoms $N$. The description by a single macroscopic wave function then breaks down, and one expects a strongly correlated ground state, such as that of an electron gas in the fractional quantum Hall regime 15, 16, 17, 18, 19. We do not address the second situation in this paper and we rather focus on the first regime. Furthermore we restrict our analysis to the case of a twodimensional gas in the $x y$ plane, assuming that a strong confinement along the $z$ direction so that the corresponding degree of freedom is frozen.

The main features of the vortex assembly equilibrium in the fast rotation regime are well known. The vortices form a triangular Abrikosov lattice in the $x y$ plane and the area of the elementary cell is $\mathcal{A}=\pi \hbar /(m \Omega)$ [20]. The atomic velocity field obtained by a coarse-grained average over a few elementary cells is equal to the rigid body rotation result $\boldsymbol{v}=\boldsymbol{\Omega} \times \boldsymbol{r}$, where $\boldsymbol{\Omega}=\Omega \hat{\boldsymbol{z}}$ ( $\hat{\boldsymbol{z}}$ is the unit vector along the $z$ axis).

Beyond this approximation, the physics is very rich and many points are still debated. In a seminal paper [9], Ho introduced the description of the macroscopic state of the rotating gas in the $x y$ plane by a wave function belonging to the Lowest Landau Level (LLL). An LLL wave function is entirely determined (up to a global phase factor) by the location of vortices. Ho considered the case of a uniform infinite vortex lattice and inferred that the ground state of the system corresponds to a gaussian shape for the coarse-grained atom density profile. Using both analytical [11, 12, 13] and numerical 14] investigations, it was subsequently pointed out that the atom distribution may have the shape of an inverted parabola, instead of Ho's gaussian result. Two paths have been proposed to explain the emergence of such non gaussian profiles. The first one assumes that the restriction to the LLL is not sufficient and the contamination of the ground state wave function by other Landau levels is responsible for the transition from a gaussian to an inverted parabola [11, 12]. The second path explores the influence of distor- 

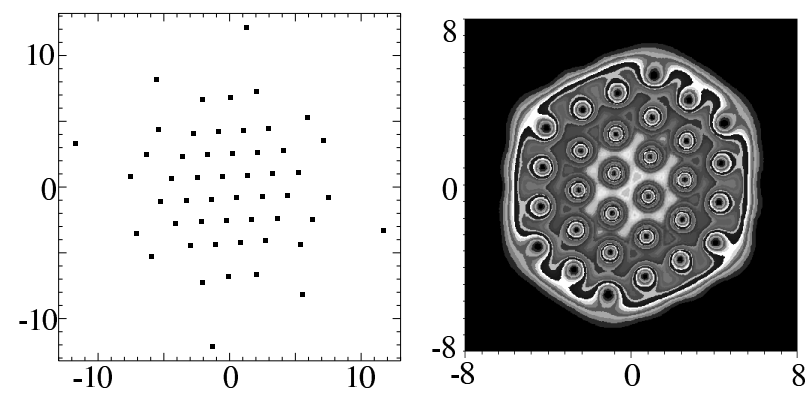

FIG. 1: Structure of the ground state of a rotating BoseEinstein condensate described by a LLL wavefunction $(\Lambda=$ 3000). (a) Vortex location; (b) Atomic density profile (with a larger scale); The reduced energy defined in Eq. (II.6) is $\epsilon=31.4107101$.

tions of the vortex lattice (within the LLL) to account for the deviation of the equilibrium profile from a gaussian [13, 14].

In the present paper, we investigate the structure of the vortex lattice for a fast rotating condensate. We derive the condition under which the LLL is a proper variational space to determine the ground state wave function within a good approximation. We present a numerical and analytical analysis of the structure of the vortex lattice, based on a minimization of the Gross-Pitaevskii energy functional within the LLL. We find that the vortices lie in a bounded domain, and that the lattice is strongly distorted on the edges of the domain. This leads to a breakdown of the rigid body rotation hypothesis which, as said above, would correspond to a uniform infinite lattice with a prescribed volume of the cell. The distortion of the vortex lattice is such that, in a harmonic potential, the coarse-grained average of the atomic density varies as an inverted parabola over the region where it takes significant values (Thomas-Fermi distribution). A similar conclusion has also been reached recently in [13, 14]. In addition to the atomic density profile, our numerical computations give access to the exact location of the zeroes of the wave function, i.e. the vortices.

An example of relevant vortex and atom distributions is shown in Fig. 11 and Fig. 10 for $n=52$ vortices. The parameters used to obtain this vortex structure correspond to a quasi-two dimensional gas of 1000 rubidium atoms, rotating in the $x y$ plane at a frequency $\Omega=0.99 \omega$, and strongly confined along the $z$ axis with a trapping frequency $\omega_{z} /(2 \pi)=150 \mathrm{~Hz}$. The spatial distribution of vortices corresponds to the triangular Abrikosov lattice only around the center of the condensate: there are about 30 vortices on the quasi-regular part of the lattice and they lie in the region where the atomic density is significant: these are the only ones seen in the density profile of Fig. 11p. At the edge of the condensate, the atomic density is reduced with respect to the central density, the vortex surface density drops down, and the vortex lattice is strongly distorted. Our analytical approach allows to justify this distortion and its relationship with the decay of the solution.

The paper is organized as follows. We start ( $($ I) with a short review of the energy levels of a single, harmonically trapped particle in a rotating frame, and we give the expression of the Landau levels for the problem of interest. Then, we consider the problem of an interacting gas in rotation, and we derive the condition for this gas to be well described by an LLL wave function ( $\S$ III). Sections III] and [V] contain the main original results of the paper. In $\S$ III we explain how to improve the determination of the ground state energy by relaxing the hypothesis of an infinite regular lattice. We present analytical estimates for an LLL wave function with a distorted vortex lattice, and we show that these estimates are in excellent agreement with the results of the numerical approach. In $\S$ IV we extend the method to non harmonic confinement, with the example of a quadratic+quartic potential. Finally we give in section $\nabla$ some conclusions and perspectives.

\section{SINGLE PARTICLE PHYSICS IN A ROTATING FRAME}

In this section, we briefly review the main results concerning the energy levels of a single particle confined in a two-dimensional isotropic harmonic potential of frequency $\omega$ in the $x y$ plane. We are interested here in the energy level structure in the frame rotating at angular frequency $\Omega(>0)$ around the $z$ axis, perpendicular to the $x y$ plane.

In the following, we choose $\omega, \hbar \omega$, and $\sqrt{\hbar /(m \omega)}$, as units of frequency, energy and length, respectively. The hamiltonian of the particle is

$$
\begin{aligned}
H_{\Omega}^{(1)} & =-\frac{1}{2} \nabla^{2}+\frac{r^{2}}{2}-\Omega L_{z} \\
& =-\frac{1}{2}(\nabla-i \boldsymbol{A})^{2}+\left(1-\Omega^{2}\right) \frac{r^{2}}{2}
\end{aligned}
$$

with $r^{2}=x^{2}+y^{2}$ and $\boldsymbol{A}=\boldsymbol{\Omega} \times \boldsymbol{r}$. This energy is the sum of three terms: kinetic energy, potential energy $r^{2} / 2$, and 'rotation energy' $-\Omega L_{z}$ corresponding to the passage in the rotating frame. The operator $L_{z}=i\left(y \partial_{x}-x \partial_{y}\right)$ is the $z$ component of the angular momentum.

\section{A. The Landau level structure}

Eq. (I.1) is formally identical to the hamiltonian of a particle of charge 1 placed in a uniform magnetic field $2 \Omega \hat{z}$, and confined in a potential with a spring constant $1-\Omega^{2}$. A common eigenbasis of $L_{z}$ and $H$ is the set of (not normalized) Hermite functions:

$$
\phi_{j, k}(\boldsymbol{r})=e^{r^{2} / 2}\left(\partial_{x}+i \partial_{y}\right)^{j}\left(\partial_{x}-i \partial_{y}\right)^{k}\left(e^{-r^{2}}\right)
$$




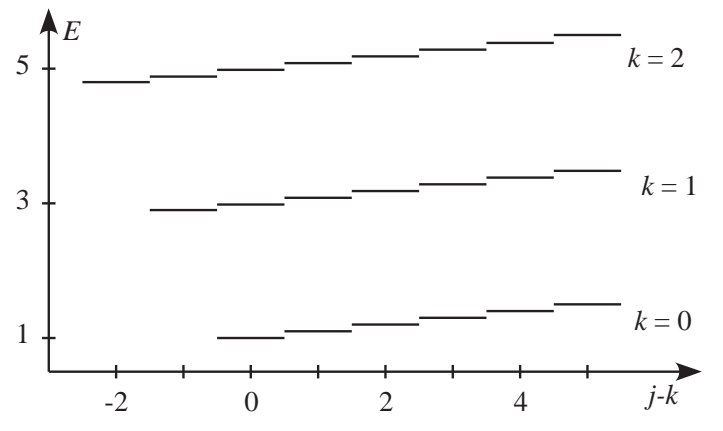

FIG. 2: Single particle spectrum for $\Omega=0.9$. The index $k$ labels the Landau levels.

where $j$ and $k$ are non-negative integers. The eigenvalues are $j-k$ for $L_{z}$ and

$$
E_{j, k}=1+(1-\Omega) j+(1+\Omega) k
$$

for $H$. For $\Omega=1$, these energy levels group in series of states with a given $k$, corresponding to the well known Landau levels. Each Landau level has an infinite degeneracy. For $\Omega$ slightly smaller than 1 , this structure in terms of Landau levels labeled by the index $k$ remains relevant, as shown in Fig. 2] The lowest energy states of two adjacent Landau levels are separated by $\sim 2$, whereas the distance between two adjacent states in a given Landau level is $1-\Omega \ll 1$.

It is clear from these considerations that the rotation frequency $\Omega$ must be chosen smaller than the trapping frequency in the $x y$ plane, i.e. $\omega=1$ with our choice of units. Otherwise the single particle spectrum Eq. (I.3) is not bounded from below. Physically, this corresponds to the requirement that the centrifugal force $m \Omega^{2} r$ must not exceed the restoring force in the $x y$ plane $-m \omega^{2} r$.

\section{B. The lowest Landau level}

When the rotation frequency $\Omega$ is close to 1 , the states of interest at low temperature are essentially those associated with $k=0$, e.g. the lowest Landau level (LLL) [9, 21, 22]. Any function $\psi(\boldsymbol{r})$ of the LLL is a linear combination of the $\phi_{j, 0}$ 's and it can be cast in the form:

$$
\psi(\boldsymbol{r})=e^{-r^{2} / 2} P(u)
$$

where $\boldsymbol{r}=(x, y), u=x+i y$ and $P(u)$ is a polynomial (or an analytic function) of $u$. When $P(u)$ is a polynomial of degree $n$, an alternative form of $\psi(\boldsymbol{r})$ is

$$
\psi(\boldsymbol{r})=e^{-r^{2} / 2} \prod_{j=1}^{n}\left(u-u_{j}\right)
$$

where the $u_{j}(j=1 \ldots n)$ are the $n$ complex zeroes of $P(u)$. Each $u_{j}$ is the position of a single-charged, positive vortex, since the phase of $\psi(\boldsymbol{r})$ changes by $2 \pi$ along a closed contour encircling $u_{j}$.
In the LLL, there is a one-to-one correspondence between atom and vortex distributions. This relation can be made explicit by introducing the atom density $\rho_{a}(\boldsymbol{r})=$ $|\psi(\boldsymbol{r})|^{2}$ :

$$
\ln \left(\rho_{a}(\boldsymbol{r})\right)=-r^{2}+2 \sum_{j} \ln \left|\boldsymbol{r}-\boldsymbol{r}_{j}\right|
$$

Introducing the vortex density $\rho_{v}(\boldsymbol{r})=\sum_{j} \delta\left(\boldsymbol{r}-\boldsymbol{r}_{j}\right)$ we obtain using $\nabla^{2}\left[\ln \left|\boldsymbol{r}-\boldsymbol{r}_{0}\right|\right]=2 \pi \delta\left(\boldsymbol{r}-\boldsymbol{r}_{0}\right)$ :

$$
\nabla^{2}\left[\ln \left(\rho_{a}(\boldsymbol{r})\right)\right]=-4+4 \pi \rho_{v}(\boldsymbol{r}) .
$$

This relation was initially derived by Ho in [9] who interpreted it in terms of the Gauss law for a system of two-dimension charges located at the points $\boldsymbol{r}_{j}$.

\section{THE INTERACTING GAS IN ROTATION}

We now consider a gas of $N$ identical bosonic atoms with mass $m$. The gas is confined in a cylindrically symmetric harmonic potential, with frequency $\omega$ in the $x y$ plane and $\omega_{z}$ along the $z$ direction. We suppose that the characteristic energy $\hbar \omega_{z}$ is very large compared to all other energy scales appearing in the paper, so that we can assume that the atoms occupy the ground state of the $z$ motion, of energy $\hbar \omega_{z} / 2$ and extension $a_{z}=\sqrt{\hbar /\left(m \omega_{z}\right)}$. We are interested in the ground state of this quasi-two dimensional gas, when it is rotating at frequency $\Omega$ close to $\omega$ around the $z$ axis.

\section{A. The Gross-Pitaevskii energy functional}

The state of the gas is described by a macroscopic wave function $\psi(\boldsymbol{r})$ normalized to unity, which minimizes the Gross-Pitaevskii energy functional. We introduce the dimensionless coefficient $G$ characterizing the strength of atomic interactions, proportional to the atom scattering length $a_{s}: G=\sqrt{8 \pi} N a_{s} / a_{z}$. The average energy per atom, written in the frame rotating at frequency $\Omega$, is:

$$
E[\psi]=\int\left(\psi^{*}\left[H_{\Omega}^{(1)} \psi\right]+\frac{G}{2}|\psi|^{4}\right) d^{2} r
$$

where $H_{\Omega}^{(1)}$ is defined in Eq. (I.1). The wave function $\psi(\boldsymbol{r})$ minimizing $E[\psi]$ satisfies the Gross-Pitaevskii equation:

$$
H_{\Omega}^{(1)} \psi(\boldsymbol{r})+G|\psi(\boldsymbol{r})|^{2} \psi(\boldsymbol{r})=\mu \psi(\boldsymbol{r})
$$

The chemical potential $\mu$ is determined by imposing that $\int|\psi|^{2}=1$. The solution of Eq. (II.2) depends on the two independent dimensionless parameters $\Omega$ and $G$. 


\section{B. The LLL limit}

In the presence of repulsive interactions $(G>0)$, the basis of Eq. (I.2) is not an eigenbasis of the $N$-body hamiltonian. However for a given interaction strength $G$ and for a sufficiently fast rotation $(\Omega$ close to $\omega=1)$, the restriction to the LLL is sufficient to determine with a good accuracy the ground state of the system and its energy. Indeed when $\Omega$ approaches $\omega=1$, the centrifugal force $m \Omega^{2} r$ nearly compensates the trapping force $-m \omega^{2} r$ and the area occupied by the atoms increases. The effect of interactions gets smaller so that the total energy per particle tends to the energy $\hbar \omega$ of the lowest Landau level, i.e. 1 in our reduced units.

When $\psi$ is chosen in the LLL, the energy functional Eq. (II.1) can be notably simplified. Indeed the LLL functions satisfy the equalities:

$$
\left\langle E_{\mathrm{kin}}\right\rangle=\left\langle E_{\mathrm{ho}}\right\rangle=\frac{1}{2}+\frac{1}{2} \int \psi^{*}\left[L_{z} \psi\right] d^{2} r
$$

where the kinetic and harmonic oscillator energies are:

$$
\left\langle E_{\mathrm{kin}}\right\rangle=\frac{1}{2} \int|\nabla \psi|^{2} d^{2} r \quad\left\langle E_{\mathrm{ho}}\right\rangle=\frac{1}{2} \int r^{2}|\psi|^{2} d^{2} r .
$$

The total energy $E[\psi]=E_{\mathrm{LLL}}[\psi]$ is then given by

$$
E_{\mathrm{LLL}}[\psi]-\Omega=\int\left((1-\Omega) r^{2}|\psi|^{2}+\frac{G}{2}|\psi|^{4}\right) d^{2} r
$$

In section [II] we will minimize this energy functional for functions in the LLL. Here we simply outline some relevant scaling laws in this regime.

\section{Scaling laws and lower bound in the LLL}

The minimization of Eq. (II.5) is equivalent to the minimization of the reduced energy

$$
\epsilon[\psi]=\frac{E_{\text {LLL }}[\psi]-\Omega}{1-\Omega}=\int\left(r^{2}|\psi|^{2}+\frac{\Lambda}{2}|\psi|^{4}\right) d^{2} r
$$

with

$$
\Lambda=\frac{G}{1-\Omega}
$$

Therefore the minimizer $\psi_{\text {LLL }}$ depends only on the parameter $\Lambda$. This is quite different from what happens when the LLL limit is not reached: for the minimization of Eq. (II.1), the two parameters $G$ and $\Omega$ are relevant, and not only their combination $\Lambda$.

It is instructive to consider the minimum of $\epsilon[\psi]$ when $\psi$ is allowed to explore the whole function space of normalized functions $\int|\psi|^{2} d^{2} r=1$. This minimum is straightforwardly obtained for $|\psi|^{2}$ varying as an inverted parabola in the disk of radius $R_{0}$ :

$$
\left|\psi_{\min }(\boldsymbol{r})\right|^{2}=\frac{2}{\pi R_{0}^{2}}\left(1-\frac{r^{2}}{R_{0}^{2}}\right), \quad R_{0}=\left(\frac{2 \Lambda}{\pi}\right)^{1 / 4}
$$

and $\psi(\boldsymbol{r})=0$ outside. The reduced energy is

$$
\epsilon_{\min }=\frac{2 \sqrt{2}}{3 \sqrt{\pi}} \sqrt{\Lambda}
$$

The variation of the atomic density as an inverted parabola is very reminiscent of the Thomas-Fermi distribution for a condensate at rest in a harmonic potential. However this analogy should be taken with care. In the usual Thomas-Fermi approach, one neglects the kinetic energy term and the equilibrium distribution is found as a balance between potential and interaction energies. In the LLL problem considered here, kinetic and potential energies are equal (see Eq. (I.3) ), and their sum $\int r^{2}|\psi|^{2}$, which is large compared to 1 when $\Lambda \gg 1$, is nearly balanced by the rotation term $-\Omega\left\langle L_{z}\right\rangle$.

The function $\psi_{\min }$ clearly does not belong to the LLL, since the only LLL function depending solely on the radial variable is $\exp \left(-r^{2} / 2\right)$. Consequently the reduced energy Eq. (11.9) is strictly lower than the result of the minimization of $\epsilon[\psi]$ with $\psi$ varying only in the LLL. In other words, the minimization of Eq. (II.5) that we perform in the next section, amounts to find the LLL function which is "the most similar" to $\psi_{\min }$, so that its reduced energy is the closest to $\epsilon_{\min }$. For $\Lambda \gg 1$, we shall see that $\epsilon \simeq \alpha \sqrt{\Lambda}$, where $\alpha$ is a coefficient of order unity to be determined.

\section{Validity of the LLL approximation}

Since $\epsilon \simeq \alpha \sqrt{\Lambda}$, the ground state energy $E$ of the fast rotating gas determined within the LLL approximation is $\Omega+\alpha \sqrt{G(1-\Omega)}$. Therefore the restriction to the LLL is valid as long as the excess energy $\alpha \sqrt{G(1-\Omega)}$ is small compared to the splitting $2 \hbar \omega=2$ between the LLL and the first excited Landau level:

$$
\text { Restriction to LLL if: } \quad G(1-\Omega) \ll 1
$$

When this condition is satisfied, the projection of $\psi$ on the excited Landau levels is negligibly small.

It is interesting to compare the scaling laws derived in the LLL with the exact relations obtained using the virial theorem. For a $2 \mathrm{D}$ gas, this theorem gives for the ground state of the (possibly rotating) system

$$
\begin{aligned}
\left\langle E_{\mathrm{ho}}\right\rangle & =\left\langle E_{\text {kin }}\right\rangle+\left\langle E_{\text {int }}\right\rangle \\
\left\langle E_{\text {int }}\right\rangle & =\frac{G}{2} \int|\psi|^{4} d^{2} r
\end{aligned}
$$

while we expect for LLL wavefunctions for $\Lambda \gg 1$

$$
\left\langle E_{\mathrm{ho}}\right\rangle=\left\langle E_{\mathrm{kin}}\right\rangle \sim \sqrt{\Lambda} \gg\left\langle E_{\mathrm{int}}\right\rangle \sim \sqrt{G(1-\Omega)} .
$$

Therefore, within the LLL validity domain of Eq. (II.10), the scaling laws for the predicted LLL energies agree with the constraints imposed by the virial theorem. 


\section{THE LLL EQUILIBRIUM DISTRIBUTION}

This section is devoted to the minimization of the reduced energy given in Eq. (II.6) for LLL wave functions. We start with wave functions corresponding to an infinite regular vortex lattice and we derive the corresponding energy. Then, we give numerical results which we use in the rest of the section as a guide to improve our choice for trial wave functions and analyze the distortion of the lattice.

\section{A. The case of a regular vortex lattice}

\section{The average density profile for a regular vortex lattice}

We consider a wave function in the LLL with an infinite number of vortices on a regular lattice and an average spatial density $\bar{\rho}_{v}$. We denote by $u_{j}$ the points of the regular triangular lattice, and by $\mathcal{A}=1 / \bar{\rho}_{v}$ the area of its unit cell. We consider the LLL wave functions

$$
\psi(\boldsymbol{r})=C e^{-r^{2} / 2} \prod_{u_{j} \in D_{R}}\left(u-u_{j}\right)
$$

where only the $u_{j}$ 's located in the disk $D_{R}$ of radius $R$ centered at the origin contribute to the product and the constant $C$ is due to the normalization $\int|\psi|^{2}=1$. For $\mathcal{A}>\pi$, we now prove the following result for the atomic density $\rho_{a}(\boldsymbol{r})=|\psi(\boldsymbol{r})|^{2}$ :

$$
\rho_{a}(\boldsymbol{r}) \rightarrow p(\boldsymbol{r}) \bar{\rho}_{a}(r) \quad \text { as } R \rightarrow \infty,
$$

where $p(\boldsymbol{r})$ is periodic over the lattice and vanishes at the $u_{j}$ 's, and where

$$
\bar{\rho}_{a}(r)=\frac{1}{\pi \sigma^{2}} e^{-r^{2} / \sigma^{2}}, \quad \frac{1}{\sigma^{2}}=1-\frac{\pi}{\mathcal{A}} .
$$

The function $\bar{\rho}_{a}(r)$ is the coarse-grained average of the atomic density $\rho_{a}(\boldsymbol{r})$. This gaussian decay has already been obtained by Ho in the so-called averaged vortex approximation 9]. However we find useful to prove it here with a different approach, which we shall generalize to non uniform lattices ( $\S$ IIC).

To prove this result, we write $\ln \left(\rho_{a}(\boldsymbol{r})\right)=v(\boldsymbol{r})+w(\boldsymbol{r})$, with $w(\boldsymbol{r})=\ln \left(\bar{\rho}_{a}(\boldsymbol{r})\right)$ and

$$
\begin{aligned}
& v(\boldsymbol{r})=\gamma^{\prime}+2 \sum_{u_{j} \in D_{R}} \ln \left|u-u_{j}\right|-\frac{2}{\mathcal{A}} \int_{P_{R}} \ln \left|u-u^{\prime}\right| d^{2} r^{\prime} \\
& w(\boldsymbol{r})=-\gamma-r^{2}+\frac{2}{\mathcal{A}} \int_{P_{R}} \ln \left|u-u^{\prime}\right| d^{2} r^{\prime},
\end{aligned}
$$

where we set $u^{\prime}=x^{\prime}+i y^{\prime}, \gamma=\ln \left(\pi \sigma^{2}\right)$ and $\gamma^{\prime}=\gamma+\ln C$. Here $P_{R}$ denotes the inner surface of the polygon formed by the union of all elementary cells having their center $u_{j}$ in the disk $D_{R}$. We want to find the limit of $v(\boldsymbol{r})$ and $w(\boldsymbol{r})$ as $R$ is large.
We start with the calculation of $v(\boldsymbol{r})$. The integral entering in the definition of $v$ can be written

$$
\frac{1}{\mathcal{A}} \int_{P_{R}} \ln \left|u-u^{\prime}\right| d^{2} r^{\prime}=\sum_{u_{j} \in D_{R}} \int \ln \left|u-u_{j}-u "\right| d^{2} r "
$$

where the sign $f$ stands for the integration over the unit cell of the lattice divided by the area of the cell $\mathcal{A}$. When $R$ tends to infinity, $v(\boldsymbol{r})$ tends to the series

$$
v_{\infty}(\boldsymbol{r})=\gamma_{0}+2 \sum_{u_{j}} \int \ln \frac{\left|u-u_{j}\right|}{\left|u-u_{j}-u^{\prime \prime}\right|} d^{2} r "
$$

whose convergence can be checked by expanding the function $\ln \left|u-u_{j}-u "\right|$ up to third order in $u " /\left(u-u_{j}\right)$. This series is a periodic function over the lattice and we set $p(\boldsymbol{r})=\exp \left(v_{\infty}(\boldsymbol{r})\right)$, which is also periodic.

To calculate $w(\boldsymbol{r})$, we first consider the auxiliary function $\tilde{w}(\boldsymbol{r})=w(\boldsymbol{r})-w(0)+r^{2} / \sigma^{2}$. Using $\nabla^{2}\left[\ln \left|\boldsymbol{r}-\boldsymbol{r}_{0}\right|\right]=$ $2 \pi \delta\left(\boldsymbol{r}-\boldsymbol{r}_{0}\right)$, we find that $\tilde{w}$ is harmonic in $P_{R}$, with $\tilde{w}(0)=0$. Moreover, a small computation leads to the inequality $\tilde{w}(\boldsymbol{r}) \geq-\pi r^{2} /(2 \mathcal{A})$. In the limit $R \rightarrow \infty$, we find that $\tilde{w}$ converges to $\tilde{w}_{\infty}$, which is a harmonic polynomial with degree less than 2 . Due to the symmetry properties of the unit cell, and the lower bound by the parabola $-\pi r^{2} /(2 \mathcal{A}), \tilde{w}_{\infty}=0$, hence the result Eq. (III.3).

To summarize, when the vortex lattice is periodic with a uniform average spatial density $\bar{\rho}_{v}$, the coarse-grain average $\bar{\rho}_{a}$ of the atomic density is the gaussian of width $\sigma$. The relation in (Eq. II.3) can be put in the form

$$
\nabla^{2}\left[\ln \left(\bar{\rho}_{a}(r)\right)\right]=-4+4 \pi \bar{\rho}_{v} .
$$

which generalizes to coarse-grained quantities the result given in Eq. (I.7). The fast rotation limit corresponds to the case of a large spatial extent of the atom distribution, i.e. $\sigma \rightarrow+\infty$ or equivalently $\mathcal{A}=1 / \bar{\rho}_{v} \rightarrow \pi$.

\section{The energy associated with a uniform vortex lattice}

Once the behavior of the limiting function $\psi$ is known, we can determine the reduced energy (II.6) in the limit of fast rotation. This requires the calculation of the integrals $\int \rho_{a}(\boldsymbol{r}) d^{2} r, \int r^{2} \rho_{a}(\boldsymbol{r}) d^{2} r$ and $\int \rho_{a}^{2}(\boldsymbol{r}) d^{2} r$ in the limit $R \rightarrow \infty$. It is performed using Eq. (III.2) and (III.3), by taking advantage on the difference in the scales of variations of $\bar{\rho}_{a}(r)($ scale $\sigma \gg 1)$ and $p(\boldsymbol{r})($ scale $\sim 1)$. We get 23]

$$
\int \rho_{a}(\boldsymbol{r}) d^{2} r \simeq\left(\oint p(\boldsymbol{r}) d^{2} r\right) \times\left(\int \bar{\rho}_{a}(r) d^{2} r\right)
$$

so that the normalization of $\rho_{a}$ entails $f p(\boldsymbol{r}) d^{2} r=1$. A similar splitting between $p$ and $\bar{\rho}_{a}$ occurs for the energy and we find:

$$
\epsilon \simeq \int\left(r^{2} \bar{\rho}_{a}(r)+\frac{b \Lambda}{2} \bar{\rho}_{a}^{2}(r)\right) d^{2} r=\sigma^{2}+\frac{b \Lambda}{4 \pi \sigma^{2}}
$$


where we have set

$$
\int p^{2}(\boldsymbol{r}) d^{2} r=b .
$$

The reduced energy Eq. (III.9) depends on the area $\mathcal{A}$ of the unit cell through $\sigma$ and on its shape through the Abrikosov coefficient $b$. Let us briefly recall the origin of this coefficient. Instead of using the exact atomic density $\rho_{a}(\boldsymbol{r})$ to calculate the energy, we work with the coarsegrain average $\bar{\rho}_{a}(r)$, whose spatial variation is much simpler. To do this substitution, we must renormalize the interaction coefficient $G$, which becomes $b G$. This is a consequence of the discreteness of the vortex distribution: since the wave function $\psi(\boldsymbol{r})$ must vanish at the vortex location, the average value of $|\psi|^{4}$ over the unit cell, hence the interaction energy, is larger than the result obtained if $|\psi|$ was quasi-uniform over the cell.

We now look for the choice of $b$ and $\sigma$ which minimizes the reduced energy Eq. (III.9). As known for the case of superconductors, the lattice minimizing $b$ is the triangular one [24], for which $b \simeq 1.1596$. The minimization over $\sigma$ then leads to:

$$
\sigma_{0}=(b \Lambda /(4 \pi))^{1 / 4} \text { and } \epsilon_{0}=\sqrt{b \Lambda / \pi} .
$$

We recover a scaling similar to Eqs. (II.8)-(II.9), inferred for a distribution varying as an inverted parabola. Note that the size of the elementary cell $\mathcal{A}=\pi\left(1-\sigma_{0}^{-2}\right)^{-1}$ differs from the rigid body rotation result, $\mathcal{A}_{\mathrm{RBR}}=\pi / \Omega$, although the two quantities tend to $\pi$ when $\Omega$ tends to 1 . Actually if we impose $\mathcal{A}=\mathcal{A}_{\mathrm{RBR}}$ in Eq. (III.9), instead of minimizing on $\sigma$, we find that $1 / \sigma^{2}=1-\Omega$ and we obtain $E_{\mathrm{LLL}} \sim 2$, much larger than the result $E_{\mathrm{LLL}} \sim 1$ deduced from Eq. (III.11).

The reduced energy $\epsilon_{0}$ exceeds the lower bound Eq. (II.9) by the factor $\sqrt{b} \times \sqrt{9 / 8} \sim 1.14$. The origin of the coefficient $b$ has been explained above. The coefficient $\sqrt{9 / 8}=1.06$ is due to the difference between the gaussian envelope found here (c.f. Eq. (III.3)), and the optimum function varying as an inverted parabola Eq. (III.8). For the parameter $\Lambda=3000$ used in Fig. 11 we find $\epsilon_{0}=33.3$ using Eq. (III.11), which is $\sim 6 \%$ larger than the result found numerically (cf. Fig. 1 and $\S$ ЩI below).

\section{B. Minimization in the LLL: numerical results}

We now turn to the description of the numerical method that has been used to obtain the vortex and atomic patterns shown in Fig. 1 and we give some further results of interest for the following discussion. For a given $\Lambda=G /(1-\Omega)$ and a given number $n$ of vortices, we write our trial functions under the form of Eq. (I.5). We vary the location of the vortices $u_{i}$ using a conjugate gradient method to determine the optimal location and the minimum reduced energy $\epsilon_{n}$.

The computation of the energy uses the Gauss point method (see 25] or 26] for a use in the case of the

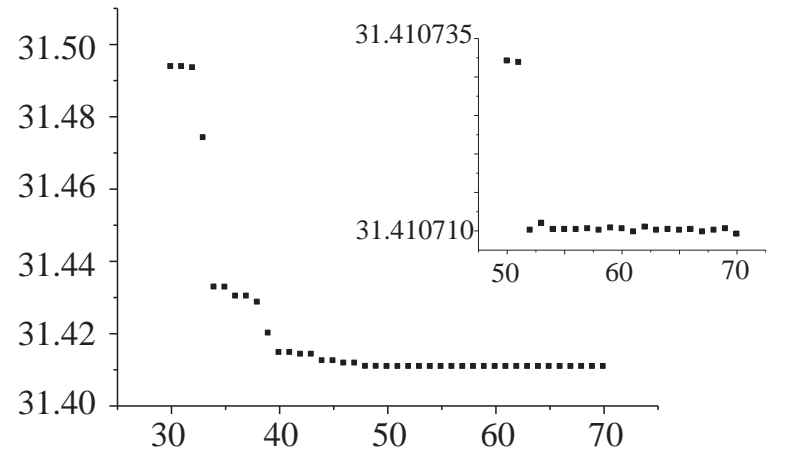

FIG. 3: Minimum reduced energy $\epsilon_{n}$ as a function of the number of vortices in the trial wave function $(\Lambda=3000)$.

Gross Pitaevski equation): the computation of the integral of a polynomial times a gaussian is exact as long as the degree of the polynomial is lower than a certain bound, which depends on the number of Gauss points. An alternative method used for example in [14] consists in writing the trial functions in the form Eq. (I.4) with $P(u)=\sum_{j=1}^{n} b_{j} u^{j}$, and performing the minimization by varying the coefficients $b_{j}$. The advantage of the method followed here is to give directly the location of the vortices, while the alternative approach requires to find the $n$ roots of the polynomial $P(u)$, which may be a delicate task for large $n$.

For the range of $\Lambda$ 's that we have explored (between 300 and 3000), the reduced energy $\epsilon_{n}$ decreases for increasing $n$, until it reaches a plateau. For $\Lambda=3000$ (Fig. 31), the plateau is reached for $n=52$ and the reduced energy varies in relative value by $\sim \pm 10^{-8}$ when $n$ increases from 52 to 70 . The vortex and atom distributions for $n=52$ are given in Fig. 1. When $n$ increases the central distribution of vortices remains the same, as well as the significant part of the atom distribution. The distribution minimizing the energy for $n=70$ vortices is shown in Fig. 4 We note that beyond $n=52$, the location of the additional vortices strongly depends on the initial data of the optimization procedure, as extra vortices only change slightly the energy. In addition to the result of Fig. 4 which is the absolute minimum for $\{\Lambda=3000, n=70\}$, we have found a number of configurations corresponding to local minima where the additional vortices lie on an outer distorted circle.

\section{The distorted lattice}

Inspired by the numerical results such as the ones shown in Fig. 1 and Fig. 团 we generalize the approach developed for the regular vortex lattice to the case of a distorted lattice. We make the hypothesis that the locations $u_{j}$ of the vortices are deduced from a regular 


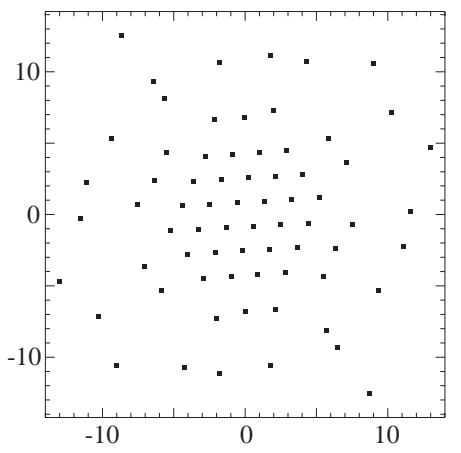

FIG. 4: Vortex distribution minimizing the reduced energy for $\Lambda=3000$ and $n=70$ vortices.

hexagonal lattice $u_{j}^{\text {reg }}$ by

$$
u_{j}=T\left(u_{j}^{\mathrm{reg}}\right)=\lambda\left(\left|u_{j}^{\mathrm{reg}}\right|\right) u_{j}^{\mathrm{reg}}
$$

where $\lambda(r)$ is a positive function varying smoothly over a distance of order unity. We assume that the unit cell of the initial regular lattice has the area $\mathcal{A}=\pi$, corresponding to a flat density profile in Eq. (III.3). If $\lambda$ tends to infinity for a finite value $r_{h}$, the number of vortices in the distorted lattice is finite and equal to $\sim r_{h}^{2}$, since all vortices located after the 'horizon' $r_{h}$ in the regular lattice are rejected to infinity. Otherwise, if $\lambda$ is finite for all $r$, the number of vortices in the distorted lattice is infinite.

The distortion is illustrated in Fig. 5] for the particular case of $\Lambda=3000$. We have plotted at the same scale the regular lattice with $\mathcal{A}=\pi$ and the configuration of vortices minimizing the energy. For $n=52$ vortices, only the lattice sites of the regular lattice whose distance to the origin is below $r_{h}=7.4$ remain in the distorted lattice. Around the center of the disk of radius $r_{h}$, the function $\lambda(r)$ is close to 1 , whereas it becomes very large when $r$ approaches the 'horizon' $r_{h}$.

As for the case of the regular lattice, we introduce the coarse-grained averages $\bar{\rho}_{a}$ and $\bar{\rho}_{v}$ of the atom and vortex densities. The function $\bar{\rho}_{v}$ is now space dependent and is simply the inverse of the area of a distorted cell in the vicinity of $\boldsymbol{r}$ :

$$
\bar{\rho}_{v}(\boldsymbol{r})=\left(\pi \lambda\left(r^{\prime}\right)\left(\lambda\left(r^{\prime}\right)+r^{\prime} \lambda^{\prime}\left(r^{\prime}\right)\right)\right)^{-1}
$$

where $\boldsymbol{r}=\lambda\left(r^{\prime}\right) \boldsymbol{r}^{\prime}$. We recall that the expected length scale in the limit of fast rotation is $R_{0}=(2 \Lambda / \pi)^{1 / 4} \gg 1$ (see $\S \amalg \mathrm{C}$ ) and we consider a class of distortions $\lambda(r)$ such that

$$
\lambda^{2}(r)=1+\frac{f\left(r^{2} / R_{0}^{2}\right)}{R_{0}^{2}}+O\left(\frac{1}{R_{0}^{4}}\right),
$$

where $f\left(\xi^{2}\right)$ is a continuous function, which diverges at $\xi_{h}^{2}=r_{h}^{2} / R_{0}^{2}$. We also assume that the integral $F(s)=$ $\int_{0}^{s} f\left(s^{\prime}\right) d s^{\prime}$ diverges at $s=\xi_{h}^{2}$. We shall check in the end that the distortion minimizing the energy belongs to the class of functions defined in Eq. (III.14).
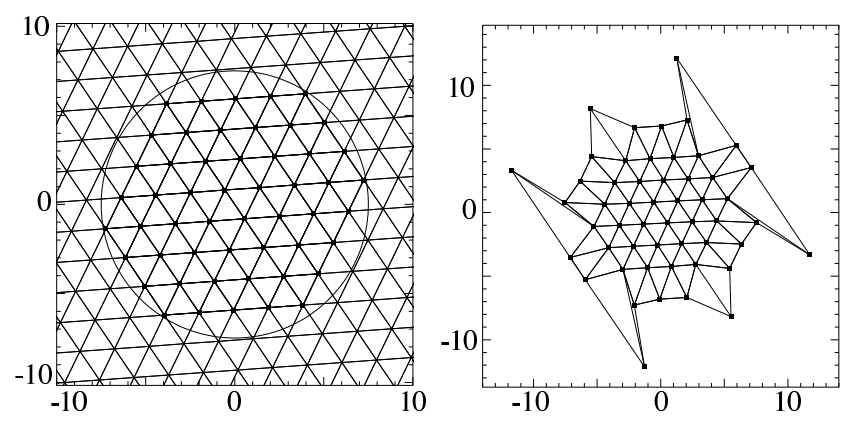

FIG. 5: Regular lattice with $\mathcal{A}=\pi$ and distorted lattice minimizing the energy for $\Lambda=3000$ and $n=52$ vortices.

In the limit $\Lambda \gg 1$, we show in the appendix the following properties for the vortex lattices obtained through a distortion obeying (Eq. III.14):

1. The atom density $\rho_{a}(\boldsymbol{r})$ can be written as

$$
\ln \left(\rho_{a}(\boldsymbol{r})\right)=v(\boldsymbol{r})+w(r)
$$

where $v$ is related to the function $v_{\infty}(\boldsymbol{r})$ introduced for a regular lattice in Eq. (III.6):

$$
v(\boldsymbol{r})=v_{\infty}\left(\boldsymbol{r}^{\prime}\right) \quad \text { with } \quad \boldsymbol{r}=\lambda\left(r^{\prime}\right) \boldsymbol{r}^{\prime} .
$$

$w(r)$ is a smooth radial function and we set $\bar{\rho}_{a}(r)=$ $\exp (w(r))$, where $\bar{\rho}_{a}$ is normalized to unity.

2. The coarse-grain average $\bar{\rho}_{a}$ at a point $\boldsymbol{r}=R_{0} \boldsymbol{\xi}$ and the integral $F$ of the distortion function $f$ are related by the relation:

$\bar{\rho}_{a}\left(R_{0} \xi\right) \propto \exp \left(-F\left(\xi^{2}\right)\right)$ if $\xi<\xi_{h}$,

and is zero elsewhere. Note that $\bar{\rho}_{a}(r)$ is continuous at $r_{h}=R_{0} \xi_{h}$ since we have assumed that $F(s)$ tends to $+\infty$ when $s \rightarrow \xi_{h}^{2}$.

3. As for the regular lattice case, we use the difference in the scales of variations of the two functions $v$ and $w$ to obtain

$$
\epsilon \simeq \int\left(r^{2} \bar{\rho}_{a}(r)+\frac{b \Lambda}{2} \bar{\rho}_{a}^{2}(r)\right) d^{2} r .
$$

where $b=1.1596$ as for a regular lattice.

The differences with respect to the initial minimization problem of Eq. (II.6) are the renormalization of the coefficient $G \rightarrow b G$ discussed in $\S \prod \mathrm{A}$ and the fact that $\bar{\rho}_{a}$ is a smooth, non-negative radial function, instead of being the square of an LLL wavefunction.

\section{The "Thomas-Fermi" distribution in the LLL}

We now address the minimization of the energy functional in Eq. (III.18). The minimizing function is the inverted parabola $\bar{\rho}_{a}(r) \propto R_{1}^{2}-r^{2}$ for $r<R_{1}=(2 b \Lambda / \pi)^{1 / 4}$, 
and $\bar{\rho}_{a}=0$ for $r>R_{1}$. The associated energy is

$$
\epsilon \simeq \frac{2 \sqrt{2}}{3 \sqrt{\pi}} \sqrt{b \Lambda}
$$

Using Eq. (III.17) we deduce the distortion function $f(s)$ and its primitive $F(s)$ :

$$
f(s) \simeq \frac{1}{\sqrt{b}-s} \quad F(s)=-\ln \left(1-\frac{s}{\sqrt{b}}\right)
$$

As initially assumed, the functions $f(s)$ and $F(s)$ tend to $+\infty$ at the horizon $\sqrt{b}$, hence $r_{h}=b^{1 / 4} R_{0}=R_{1}$. This means that at leading order in $\Lambda$, the Thomas Fermi radius and the horizon are equal. The function $T$ transforming the initial regular lattice $u_{j}^{\text {reg }}$ into the distorted lattice $u_{j}$ is thus:

$$
\boldsymbol{r}=T\left(\boldsymbol{r}^{\prime}\right)=\boldsymbol{r}^{\prime}+\frac{\boldsymbol{r}^{\prime}}{R_{1}^{2}-r^{\prime 2}} .
$$

Once $f(s)$ is known, one can evaluate the vortex density using Eq. (111.13):

$$
\pi \bar{\rho}_{v}(r)=\left(1+\frac{R_{1}^{2}}{\left(R_{1}^{2}-r^{\prime 2}\right)^{2}}\right)^{-1} .
$$

where $\boldsymbol{r}$ and $\boldsymbol{r}^{\prime}$ are related by Eq. (III.21). In particular the vortex density at $r=0$ is $\sim\left(1-R_{1}^{-2}\right) / \pi$, which is close, but not equal, to the prediction $\Omega / \pi$ for a rigid body rotation.

Our distortion function $f$ is to be related to that of [28], though it is derived using very different techniques. The asymptotic result Eq. (III.19) has also been obtained recently by Watanabe, Baym and Pethick 13. who assumed that Eq. (III.7) can be generalized to the case where $\bar{\rho}_{v}$ is spatially dependent:

$$
\nabla^{2}\left[\ln \left(\bar{\rho}_{a}(r)\right)\right]=-4+4 \pi \bar{\rho}_{v}(r) .
$$

By differentiating Eq. (III.17), a similar relation can be proved within our approach with $\bar{\rho}_{v}(r)$ replaced by $\bar{\rho}_{v}(T(r))$. The two relations are equivalent at points not too close to the Thomas-Fermi radius (i.e. $R_{1}-r \gtrsim 1$ ). A result related to Eq. (III.23) has also been shown in a different context by Sheehy and Radzihovsky [27]. They consider the case of a condensate which is not in very fast rotation (i.e. outside of the LLL regime) but still with several vortices. Interestingly, the procedure used in [27] to derive the relation between $\bar{\rho}_{v}$ and $\bar{\rho}_{a}$ is based on the minimization of the energy functional, including atom interactions. On the contrary, the result in Eq. (III.17) or Eq. (III.23) is a consequence of the structure of an LLL wave function and it is at first sight independent of atomic interactions. However one must keep in mind that the knowledge of the strength of atom interactions is essential to check the relevance of LLL wave functions for the problem (see Eq. (II.10)). The relation reached in [27] has the same structure as Eq. (III.23), but with a

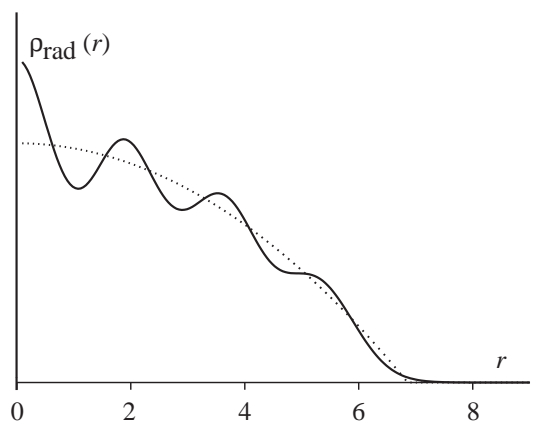

FIG. 6: Radial density distribution $\rho_{\text {rad }}(r)$ for $\Lambda=3000$. The unit along the vertical direction is arbitrary. The dotted line is a fit using the inverted parabola with the radius $R_{1}=$ $(2 b \Lambda / \pi)^{1 / 4}$, with an adjustable amplitude.

dimensionless coefficient involving the healing length and $\bar{\rho}_{v}$ inside the $\nabla^{2} \ln \left(\rho_{a}\right)$ term. Close to the Thomas-Fermi radius, $\bar{\rho}_{v}$ varies rapidly and the approach of [27] leads to a different relation from Eq. (III.23), since the derivatives of $\bar{\rho}_{v}$ have a significant contribution in this region.

Our analytical predictions can be compared with our numerical results obtained in the particular case $\Lambda=$ 3000 (i.e. $R_{1}=6.86$ ), for which we plotted Fig. 1] The prediction of Eq. (III.19) yields $\epsilon=31.374$, only $0.12 \%$ below the value determined numerically. We can also compare our trial density with the numerical result. We give in Fig. 6 the prediction of the inverted parabola together with the radial density distribution determined numerically:

$$
\rho_{\mathrm{rad}}(r)=\frac{1}{2 \pi} \int_{0}^{2 \pi}|\psi(\boldsymbol{r})|^{2} d \theta
$$

where $\theta$ is the polar angle in the $x y$ plane. Apart from oscillations due to the discreteness of vortices, the two distributions are remarkably close to each other. A similar conclusion was reached recently by Cooper, Komineas, and Read 14. They also performed a numerical minimization of the energy of Eq. (II.1) in the LLL limit, and found an atom density profile in good agreement with the inverted parabola distribution predicted in [13].

From the above analytical results, we expect that the minimizing configuration will involve $n \sim r_{h}^{2} \sim 48$ vortices. The number of vortices for which the minimum energy plateau is reached numerically is 52 , which is very close to $r_{h}^{2}$. As for the location of vortices, our analysis indicates that the vortices in the distorted lattice are images through Eqs. (III.12)-(III.21) of points of the regular hexagonal lattice such that $\left|u_{j}^{\text {reg }}\right|<r_{h}=R_{1}$. Note that the optimal vortex configuration involves some vortices outside the disk of radius $R_{1}$. They correspond to regular lattice sites $\left|u_{j}^{\mathrm{reg}}\right|$ close to the horizon $r_{h}$. Indeed, for these points $\lambda$ gets large and the image point is sent beyond the Thomas Fermi radius. Thus, though the distorted lattice provides an inverted parabola which 
vanishes at $R_{1}$, the location of the vortices extends beyond $R_{1}$. The numerical analysis leads to results which nicely confirm our analytical predictions. In addition it allows to explore the role of the vortices lying outside the Thomas-Fermi distribution. For example one can remove the contribution $\left(u-u_{j}\right)$ of these vortices in the expression Eq. (I.5) of the LLL wave function, while keeping unchanged the contribution of the vortices inside the Thomas-Fermi radius. This results in a significant modification of $\rho_{a}(\boldsymbol{r})$ which then vanishes around $\sim 7.3$, instead of $\sim 6.8$. Therefore these outer vortices play an important role in the equilibrium shape of the condensate, even though they cannot be found when one simply plots the atomic spatial density.

A closer look at Fig. [6 indicates that $\bar{\rho}_{a}$ is matched to zero more smoothly than an inverted parabola. An expansion of the energy of the distorted lattice (Eq. (III.18) ) to the next order in $\Lambda$ should lead to a minimizing function $\bar{\rho}_{a}$ with a smoother decay to zero around $R_{1}$. In particular a natural way to match the inverted parabola with the asymptotic decay $r^{2 n} e^{-r^{2}}$ of any LLL function with $n$ vortices, could be obtained through a Painlevé-type equation (as at the border of a non-rotating BEC).

Remark: Comparison with the "centrifugal force approximation". Under some conditions, it is possible to write $\psi$ as the product of a rapidly varying function $\eta(\boldsymbol{r})$ and a slowly varying envelope $\bar{\psi}(\boldsymbol{r})[11]$. This is reminiscent of the splitting of $\ln \left(\rho_{a}\right)$ in terms of $v$ and $w$, although it leads to a different conclusion. One obtains for the envelope an equation similar to Eq. (II.2), where only the centrifugal potential remains [1]]:

$$
-\frac{1}{2} \nabla^{2} \bar{\psi}(\boldsymbol{r})+\left(1-\Omega^{2}\right) \frac{r^{2}}{2} \bar{\psi}(\boldsymbol{r})+G|\bar{\psi}(\boldsymbol{r})|^{2} \bar{\psi}(\boldsymbol{r})=\bar{\mu} \bar{\psi}(\boldsymbol{r})
$$

where $\bar{\mu}=\mu-\Omega$. We call this approach the "centrifugal force" approximation [29] and we compare its predictions with those derived from the LLL approximation.

When the approximation leading to Eq. (III.25) is valid, one is left with the problem of a $2 \mathrm{D}$ gas at rest in a harmonic potential with the spring constant $1-\Omega^{2}$. The solution of this equation depends on the strength of the interaction parameter $G$. If $G \gg 1$, the kinetic energy term can be neglected (Thomas-Fermi approximation) and one gets $|\bar{\psi}(\boldsymbol{r})|^{2} \propto 1-r^{2} / R_{\mathrm{cfa}}^{2}$ inside the disk of radius $R_{\text {cfa }}=\left(4 G /\left[\pi\left(1-\Omega^{2}\right)\right]\right)^{1 / 4}$ and $\psi(\boldsymbol{r})=0$ outside. Note that $R_{\text {cfa }}$ coincides with our Thomas-Fermi radius $R_{1}$ for $\Omega \simeq 1$. If $G \ll 1$, the interaction term can be neglected and the solution is the ground state of the harmonic oscillator, i.e. the gaussian of width $\left(1-\Omega^{2}\right)^{-1 / 4}$.

In the LLL, we have seen that the distinction between the two regimes $G \gg 1$ and $G \ll 1$ is not relevant. The only important parameter is $\Lambda=G /(1-\Omega)$. When $\Lambda \gg 1$ the envelope of the atom density profile is close to an inverted parabola, irrespective of the value of $G$. Therefore, there exists a clear discrepancy between the predictions of the LLL treatment and those of the centrifugal force ap- proximation when $1-\Omega \ll G \ll 1$. For these parameters the LLL approximation is valid since $G(1-\Omega) \ll 1$ (see Eq. (II.10). The extent of the wave function minimizing $\epsilon[\psi]$ is thus $R_{1} \sim(G /(1-\Omega))^{1 / 4}$, while the reasoning based on Eq. (III.25 would lead to a gaussian envelope with a larger size $(1-\Omega)^{-1 / 4}$, independent of $G$.

\section{EXTENSION TO OTHER CONFINING POTENTIALS}

The ideas that we have developed for a harmonic confinement can be generalized to a larger class of trapping potential $V(\boldsymbol{r})$. For simplicity we assume here that $V$ is cylindrical symmetric, with a minimum at $r=0$. We define $\omega$ as $m \omega^{2}=\partial^{2} V /\left.\partial r^{2}\right|_{0}$ and we set

$$
V(r)=\frac{1}{2} m \omega^{2} r^{2}+W(r) \text {. }
$$

As above we choose $\omega$ and $\sqrt{\hbar /(m \omega)}$ as the units for frequency and length, respectively.

We are still interested here in a region where $\Omega \sim 1$. To minimize the Gross-Pitaevskii energy functional, we use again wave functions in the LLL so that the energy per particle to be minimized is

$$
E_{\mathrm{LLL}}=\Omega+\int\left[\left((1-\Omega) r^{2}+W(r)\right) \rho_{a}+\frac{G}{2} \rho_{a}^{2}\right] d^{2} r
$$

As explained in section II.C, the LLL approximation is valid if the minimum for $E_{\mathrm{LLL}}-\Omega$ is small compared to the distance $2=2 \hbar \omega$ between the LLL and the first excited Landau level.

We have seen that varying the locations $u_{i}$ of the vortices, hence the average vortex surface density $\bar{\rho}_{v}$, allows to generate a large class of coarse-grain averaged atom densities $\bar{\rho}_{a}$. Provided $W(r)$ is well behaved, we can generalize the treatment presented for the purely quadratic case. The energy $E_{\mathrm{LLL}}$ can still be expressed in terms of $\bar{\rho}_{a}$ instead of $\rho_{a}$ with an expression similar to Eq. (IV.2), and the interaction parameter $G$ replaced by $b G$. The Thomas-Fermi distribution minimizing $E_{\mathrm{LLL}}$ is

$$
\rho_{a}^{\mathrm{TF}}(r)=\max \left(\frac{\mu-(1-\Omega) r^{2}-W(r)}{b G}, 0\right)
$$

where $\mu$ is the chemical potential determined such that $\int \bar{\rho}_{a}=1$. Once $\bar{\rho}_{a}$ has been determined over the whole space, the energy $E_{\mathrm{LLL}}$ can then be calculated and the validity of the various approximations can be checked: (i) $\left|E_{\mathrm{LLL}}-\Omega\right| \ll 1$ and (ii) the extension of the domain where $\bar{\rho}_{a}$ differs from zero is large compared to 1 , so that it is legitimate to introduce a coarse-grain average of $\rho_{a}$ over several vortex cells, and there is a large parameter playing the role of $R_{0}$.

As an example, we investigate the case of a combined quartic and harmonic potential: $W(r)=k r^{4} / 4$, which has been studied recently both theoretically, numerically 
30, 31, 32, 33, 34, 35, 36] and experimentally [37]. A nice feature of this potential is that it allows to explore the region $\Omega \geq 1$, since the centrifugal force, $-\Omega^{2} r$, can always be compensated by the trapping force, varying as $-\left(r+k r^{3}\right)$. We define $\Delta_{0}=\left(3 k^{2} b G /(8 \pi)\right)^{2 / 3}$ and $\Delta=(1-\Omega)^{2}+k \mu$. Two cases can occur. (i) If $\Omega<$ $\Omega_{c}=1+\sqrt{\Delta_{0}}$, then $\rho_{a}^{\mathrm{TF}}$ is non zero in a disc of radius $R_{+}^{2}=2(\Omega-1+\sqrt{\Delta}) / k, \Omega$ and $\Delta$ being linked by

$$
2 \Delta^{3 / 2}+3 \Delta(\Omega-1)-(\Omega-1)^{3}=4 \Delta_{0}^{3 / 2} .
$$

(ii) If $\Omega>\Omega_{c}$, then $\rho_{a}^{\mathrm{TF}}$ is non-zero on an annulus of radii $R_{ \pm}^{2}=2(\Omega-1 \pm \sqrt{\Delta}) / k$, and $\Delta=\Delta_{0}$.

The Thomas-Fermi distribution given in Eq. (IV.3) allows to calculate the minimum energy per particle. Since the general calculation is quite involved, we simply give here the result for $\Omega=\Omega_{c}$ :

$$
\Omega=\Omega_{c}: \quad E_{\mathrm{LLL}}-\Omega=\alpha k^{1 / 3} G^{2 / 3}
$$

where $\alpha \simeq-0.1$. More generally, when $|1-\Omega|$ is at most of the order of $k^{2 / 3} G^{1 / 3}$, then $E_{\mathrm{LLL}}-\Omega$ is of the order of $k^{1 / 3} G^{2 / 3}$. The restriction to the LLL wave functions and the use of the 'Thomas-Fermi' approximation (Eq. (IV.3) are valid if two conditions are fulfilled: (i) $E_{\mathrm{LLL}}-\Omega \ll$ 1 , hence $k G^{2} \ll 1$, (ii) the extension $R_{+} \sim(G / k)^{1 / 6}$ of $\bar{\rho}_{a}$ is large compared to 1 , so that the coarse-grain average of $\rho_{a}$ is meaningful. This requires $k \ll 1$ and $k \ll G \ll 1 / \sqrt{k}$. When these conditions are satisfied, $\Omega_{c}-1 \sim k^{2 / 3} G^{1 / 3} \ll 1$, and the study of the regime $\Omega \geq \Omega_{c}$ can be performed within the LLL. In addition one can check that for $\Omega_{c}-1<\Omega-1 \ll k^{1 / 3} G^{2 / 3}$, the width $R_{+}-R_{-}$of the annulus is large compared to 1 (both $R_{+}$and $R_{-}$are of order $(G / k)^{1 / 6}$ ), so that the use of the coarse-grain averages of $\rho_{a}$ and $\rho_{v}$ is justified. A similar analysis to what we have performed above yields an almost uniform vortex lattice in the annulus, with a distortion near the inner and outer boundaries.

The LLL approximation has been used by Jackson, Kavoulakis and Lundh to study the phase diagram of the vortices in a quadratic+quartic phase [35]. They were mostly interested in the stability of giant vortices, hence they restricted their analysis to particular LLL states, where $F(u)$ only contains two or three terms $b_{j} u^{j}$. However one could in principle use the same approach as the numerical treatment developed here, and derive the detailed vortex pattern for various choices of $G, \Omega$ and $k$. It would be interesting to see whether there exists a domain of parameters where the polynomial $F(u)$ has a multiple root in $u=0$. This would correspond to the giant vortex which has been predicted by other approaches [10, 32, 36]. Another limit where $R_{+}-R_{-} \leq 1$ has recently been studied in 36 .

\section{CONCLUSION}

In this paper, we have studied analytically and numerically the vortex distribution and atomic density for the ground state of a rotating condensate trapped in a harmonic potential, when the rotation and trapping frequencies are close to each other. Restricting our analysis to quantum states in the lowest Landau level, we have shown that the atomic density varies as an inverted parabola over a central region. The vortices form an almost regular triangular lattice in this region, but the area of the cell differs from the prediction for solid body rotation. In the outer region, the lattice is strongly distorted. We have determined the optimal distortion, and related it to the decay of the wave function close to the Thomas-Fermi radius.

Our results agree with those of a recent numerical study [14]. Another analytical approach to this problem has recently been given in [13]. It leads to the same value as ours for the energy of the ground state, whereas our treatment provides more detailed information on the vortex pattern at the edge of the condensate. Our predictions for the equilibrium shape of the atomic density and for the vortex distribution should be experimentally testable. In [7] the regime of fast rotation in the LLL has already been achieved and it was indeed found that the atom density profile varies as an inverted parabola, and not as a Gaussian as one would expect for an infinite regular lattice [9]. In [8], a detailed experimental analysis of the vortex spacing as a function of the distance to the center of the trap has been made and it showed a clear distortion of the pattern on the edges of the condensate. This study was not performed in conditions such that our LLL approximation is valid, and the relevant theoretical model is rather the one developed in 27]. However it should be possible to perform a similar experimental analysis for faster rotation rates, and test in particular the validity of our prediction concerning the distortion factor $\lambda(r)$ (see Eqs. (III.12)-(III.14)-(III.20) ).

Finally, we have addressed the case of other trapping potentials, such as a superposition of a quadratic and a quartic potential, which have also been addressed experimentally [37]. For even faster rotations, when the number of vortices approaches the number of atoms, the ground state is strongly correlated. We did not touch this point here, but our work should be relevant for studying the apparition of this correlated regime from a destabilization of the mean field results by quantum fluctuations.

\section{Acknowledgments}

J.D. is indebted to Yvan Castin and Vincent Bretin for several insightful discussions. A.A. and X.B. are very grateful to Françoit Murat for explaining details on homogeneisation techniques and to Eric Cancès for pointing out the method of Gauss points in the computations. This work is partially supported by the fund of the French ministry for research, ACI "Nouvelles interfaces des mathématiques", CNRS, Collège de France, Région Ile de France, and DRED. 


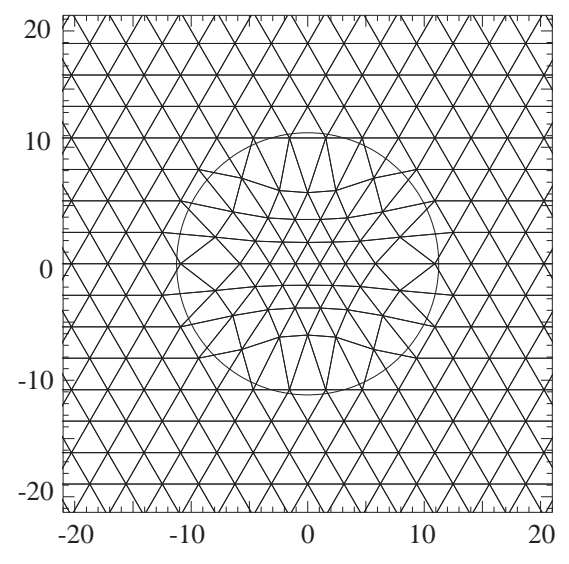

FIG. 7: Example of a distorted lattice generated by the transformation Eq. (VI.1). The radius of the circle is $\lambda_{\alpha} \alpha R_{0}$. In the regular part outside the circle, the cell area is $\mathcal{A}_{\alpha}$.

\section{APPENDIX}

The aim of this appendix is to prove the properties used in $\S$ IIC A detailed proof will be given in 38]. We consider a distorted lattice in an inner region and keep a regular lattice in the outer region in such a way that the distortion is continuous (see Fig. 7). We label by $\boldsymbol{j}$ a regular hexagonal lattice with a unit cell area $\mathcal{A}=\pi$ and we define the transformed lattice by

$$
u_{j}=\left\{\begin{array}{lll}
\lambda(|\boldsymbol{j}|) \boldsymbol{j} & \text { for } & |\boldsymbol{j}|<\alpha R_{0} \\
\lambda_{\alpha} \boldsymbol{j} & \text { for } & |\boldsymbol{j}| \geq \alpha R_{0}
\end{array}\right.
$$

where the radius $R_{0}$ is given in Eq. (II.8), the distortion function $\lambda(r)$ satisfies (III.14), and $\alpha R_{0}$ is smaller than the horizon $r_{h}$ where $\lambda(r)$ diverges. We have set $\lambda_{\alpha}=$ $\lambda\left(\alpha R_{0}\right)$ and the area of the unit cell of the outer lattice is $\mathcal{A}_{\alpha}=\pi \lambda_{\alpha}^{2}$. When $\alpha R_{0}$ tends to $r_{h}, \mathcal{A}_{\alpha}$ tends to infinity and the vortex lattice of Fig. [7 is similar to the one in the right of Fig. 5

In the following, we shall (i) define $\bar{\rho}_{a}$ and compute its limit when $R_{0}$ increases (i.e. $\Omega$ tends to 1 ) for a fixed $\alpha$, (ii) let $\alpha$ get close to the horizon $\xi_{h}=r_{h} / R_{0}$. We need to take the limits in this order, because we will use that $\lambda_{\alpha}$ is close to 1 , which is only true if $\alpha$ is fixed less than $\xi_{h}$ and $R_{0}$ is large.

Firstly we consider only the points $\boldsymbol{j}$ in a disc $D_{R^{\prime}}$

$$
\psi(\boldsymbol{r})=C e^{-r^{2} / 2} \prod_{|\boldsymbol{j}|<R^{\prime}}\left(u-u_{j}\right)
$$

$Q_{\alpha}$ denotes the unit cell of the lattice of area $\mathcal{A}_{\alpha}$ and $P_{\alpha, R^{\prime}}$ is the polygon formed by the union of all elementary cells of area $\mathcal{A}_{\alpha}$ and center $\lambda_{\alpha} \boldsymbol{j}$, with $|\boldsymbol{j}|<R^{\prime}$. We write $\ln \left(\rho_{a}(\boldsymbol{r})\right)=v_{R^{\prime}}(\boldsymbol{r})+w_{R^{\prime}}(\boldsymbol{r})$ with,

$$
\begin{aligned}
v_{R^{\prime}}(\boldsymbol{r}) & =2 \sum_{\alpha R_{0}<|\boldsymbol{j}|<R^{\prime}}\left(\ln \left|\boldsymbol{r}-\lambda_{\alpha} \boldsymbol{j}\right|\right. \\
& \left.-\frac{1}{\mathcal{A}_{\alpha}} \int_{Q_{\alpha}} \ln \left|\boldsymbol{r}-\boldsymbol{r}^{\prime}-\lambda_{\alpha} \boldsymbol{j}\right| d^{2} r^{\prime}\right) \\
& +2 \sum_{|\boldsymbol{j}|<\alpha R_{0}}(\ln |\boldsymbol{r}-\lambda(\boldsymbol{j}) \boldsymbol{j}| \\
& \left.-\frac{1}{\mathcal{A}_{\alpha}} \int_{Q_{\alpha}} \ln \left|\boldsymbol{r}-\boldsymbol{r}^{\prime}-\lambda(\boldsymbol{j}) \boldsymbol{j}\right| d^{2} r^{\prime}\right)
\end{aligned}
$$

and $w_{R^{\prime}}(\boldsymbol{r})=w_{1 R^{\prime}}(\boldsymbol{r})+w_{2}(\boldsymbol{r})$ with

$$
\begin{aligned}
w_{1 R^{\prime}}(\boldsymbol{r}) & =-r^{2}+\frac{2}{\mathcal{A}_{\alpha}} \int_{P_{\alpha, R^{\prime}}} \ln \left|\boldsymbol{r}-\boldsymbol{r}^{\prime}\right| d^{2} r^{\prime} \\
w_{2}(\boldsymbol{r}) & =\sum_{|\boldsymbol{j}|<\alpha R_{0}} \frac{2}{\mathcal{A}_{\alpha}} \int_{Q_{\alpha}} \ln \frac{\left|\boldsymbol{r}-\boldsymbol{r}^{\prime}-\lambda(\boldsymbol{j}) \boldsymbol{j}\right|}{\left|\boldsymbol{r}-\boldsymbol{r}^{\prime}-\lambda_{\alpha} \boldsymbol{j}\right|} d^{2} r^{\prime}
\end{aligned}
$$

We have just added and subtracted terms at this stage.

Now we let $R^{\prime}$ tend to infinity and find the limit for an infinite number of vortices. This step is very similar to the case of the regular lattice since the lattice distortion only affects a finite number of sites. We find that

$$
w_{1 R^{\prime}}(\boldsymbol{r})-w_{1 R^{\prime}}(0) \rightarrow w_{1}(\boldsymbol{r})=-r^{2} / \sigma^{2}
$$

with $\sigma^{-2}=1-\pi / \mathcal{A}_{\alpha} \cdot v_{R^{\prime}}$ tends to a convergent series $v$ (which is not a periodic function, contrary to the regular lattice case).

The next step is to let $R_{0}$ be large, keeping $\alpha$ fixed, so that $\lambda_{\alpha}$ is close to 1 for the class of distortion functions considered in Eq. (III.14). We find

$$
v(\boldsymbol{r}) \simeq v_{\infty}\left(\boldsymbol{r}^{\prime}\right)
$$

where $v_{\infty}$ is given by Eq. (III.6), and $\boldsymbol{r}, \boldsymbol{r}^{\prime}$ are related by

$$
\boldsymbol{r}= \begin{cases}\lambda\left(r^{\prime}\right) \boldsymbol{r}^{\prime} & \text { for } r / \lambda_{\alpha} \leq \alpha R_{0} \\ \lambda_{\alpha} \boldsymbol{r}^{\prime} & \text { for } r / \lambda_{\alpha}>\alpha R_{0},\end{cases}
$$

We denote $w=w_{1}+w_{2}=\ln \left(\bar{\rho}_{a}\right)$. We estimate $w_{2}(r)$, using an expansion of the logarithm and the fact that $\lambda(\boldsymbol{j}) \sim \lambda_{\alpha} \sim 1$ :

$$
w_{2}\left(R_{0} \boldsymbol{\xi}\right) \simeq \frac{1}{\pi} \int_{\xi^{\prime}<\alpha}\left(f\left(\alpha^{2}\right)-f\left(\xi^{\prime 2}\right)\right) \frac{\boldsymbol{\xi}^{\prime} \cdot\left(\boldsymbol{\xi}-\boldsymbol{\xi}^{\prime}\right)}{\left|\boldsymbol{\xi}-\boldsymbol{\xi}^{\prime}\right|^{2}} d^{2} \xi^{\prime} .
$$

where relevant $\xi$ 's are of order unity. Using an integration by part and a primitive $F$ of $f$, we get $(\theta(x)$ is the Heaviside function):

$$
w_{2}\left(R_{0} \boldsymbol{\xi}\right) \simeq\left[F\left(\alpha^{2}\right)-F\left(\xi^{2}\right)+\left(\xi^{2}-\alpha^{2}\right) f\left(\alpha^{2}\right)\right] \theta(\alpha-\xi)
$$

Since we have $\sigma^{-2} \simeq f\left(\alpha^{2}\right) / R_{0}^{2}$, then $w_{1}\left(R_{0} \boldsymbol{\xi}\right)=$ $-\xi^{2} f\left(\alpha^{2}\right)$. Putting everything together, we obtain up to an additive constant for normalization,

$$
\ln \left(\bar{\rho}_{a}\left(R_{0} \boldsymbol{\xi}\right)\right) \simeq \begin{cases}-F\left(\xi^{2}\right) & \text { for } \quad \xi<\alpha \\ -\xi^{2} f\left(\alpha^{2}\right)+\mu & \text { for } \quad \xi>\alpha\end{cases}
$$


with $\mu=\alpha^{2} f\left(\alpha^{2}\right)-F\left(\alpha^{2}\right)$.

Finally, using that $\lambda_{\alpha} \simeq 1$, we can apply the separation of integrals 23] and find for example that

$$
\begin{aligned}
\int \rho_{a} d^{2} r \propto & \left(\int e^{v_{\infty}\left(\boldsymbol{r}^{\prime}\right)} d^{2} r^{\prime}\right) \times \\
& \left(\int_{\xi<\alpha} e^{-F\left(\xi^{2}\right)} d^{2} \xi+e^{\mu} \int_{\xi>\alpha} e^{-\xi^{2} f\left(\alpha^{2}\right)} d^{2} \xi\right)
\end{aligned}
$$

The last integral in the second line is equal to $\pi e^{-F\left(\alpha^{2}\right)} / f\left(\alpha^{2}\right)$. At this stage, $\alpha$ is still a free parameter. If the distortion function $\lambda(r)$ has a horizon at $r=\xi_{h} R_{0}$, we let $\alpha$ tend to $\xi_{h}$, otherwise to $\infty$. The last integral tends to zero given the hypothesis that $f$ and $F$ tend to $+\infty$ at $\xi_{h}$. The same procedure is valid for all terms entering into the energy functional, which justifies the use of Eq. (III.18).
[1] E. M. Lifshitz and L. P. Pitaevskii, Statistical Physics, Part 2, chap. III (Butterworth-Heinemann, 1980).

[2] R. J. Donnelly, Quantized Vortices in Helium II, (Cambridge, 1991), Chaps. 4 and 5.

[3] M. R. Matthews et al., Phys. Rev. Lett. 83, 2498 (1999).

[4] K. W. Madison, F. Chevy, W. Wohlleben, and J. Dalibard, Phys. Rev. Lett. 84, 806, (2000).

[5] J. R. Abo-Shaeer, C. Raman, J. M Vogels, and W. Ketterle, Science 292, 476 (2001); C. Raman, J. R. AboShaeer, J. M. Vogels, K.Xu, and W. Ketterle, Phys. Rev. Lett. 87, 210402 (2001).

[6] P. Engels, I. Coddington, P. C. Haljan, V. Schweikhard, and E. A. Cornell, Phys. Rev. Lett. 90, 170405 (2003).

[7] V. Schweikhard, I. Coddington, P. Engels, V. P. Mogendorff, and E. A. Cornell, Phys. Rev. Lett. 92, 040404 (2004).

[8] I. Coddington, P. C. Haljan, P. Engels, V. Schweikhard, S. Tung, E. A. Cornell, cond-mat/0405240

[9] T. L. Ho, Phys. Rev. Lett. 87, 060403 (2001).

[10] U. R. Fischer and G. Baym, Phys. Rev. Lett. 90, 140402 (2003).

[11] G. Baym and C. J. Pethick, Phys. Rev. A 69, 043619(2004).

[12] G. Watanabe and C. J. Pethick, cond-mat/0402167

[13] G. Watanabe, G. Baym and C. J. Pethick, cond-mat/0403470

[14] N. R. Cooper, S. Komineas and N. Read, Phys. Rev. A 70, 033604 (2004) .

[15] N. R. Cooper, N. K. Wilkin, and J. M. F. Gunn, Phys. Rev. Lett. 87, 120405 (2001).

[16] B. Paredes, P. Fedichev, J. I. Cirac, and P. Zoller, Phys. Rev. Lett. 87, 010402 (2001).

[17] J. Sinova, C. B. Hanna, and A. H. MacDonald, Phys. Rev. Lett. 89, 030403 (2002).

[18] J. W. Reijnders, F. J. M. van Lankvelt, K. Schoutens, and N. Read, Phys. Rev. Lett. 89, 120401 (2002)

[19] N. Regnault and T. Jolicoeur, Phys. Rev. Lett. 91,
$030402(2003)$.

[20] R. P. Feynman, in Progress in Low Temperature Physics, vol. 1, Chapter 2, C.J. Gorter Ed. (North-Holland, Amsterdam, 1955).

[21] S. M. Girvin and T. Jach, Phys. Rev. B 29, 5617 (1984).

[22] D. A. Butts and D. S. Rokhsar, Nature 397, 327 (1999).

[23] G. Allaire, SIAM J. Math. Anal. 23 1482-1518 (1992).

[24] W. H. Kleiner, L. M. Roth and S. H. Autler, Phys. Rev. 133, A1226, (1964).

[25] Y. Maday and C. Bernardi, in Handbook of numerical analysis, vol. 5, 209-486, Elsevier, Amsterdam, (2000).

[26] C. M. Dion and E. Cancès, Phys. Rev. E 67, 046706 (2003).

[27] D. E. Sheehy and L. Radzihovsky, cond-mat/0402637 and cond-mat/0406205

[28] J. Anglin and M. Crescimanno, cond-mat/0210063

[29] Strictly speaking, as shown in 11], the coefficient $G$ is renormalized in this procedure. Here we omit this change for our qualitative discussion.

[30] A. L. Fetter, Phys. Rev. A 64, 063608 (2001).

[31] K. Kasamatsu, M. Tsubota, and M. Ueda, Phys. Rev. A 66, 053606 (2002).

[32] E. Lundh Phys. Rev. A 65, 043604 (2002).

[33] G. M. Kavoulakis and G. Baym, New Jour. Phys. 5, 51.1 (2003).

[34] A. Aftalion and I. Danaila, Phys. Rev. A 69, 033608 (2004).

[35] A. D. Jackson, G. M. Kavoulakis, and E. Lundh, Phys. Rev. A 69, 053619 (2004); see also A. D. Jackson and G. M. Kavoulakis, cond-mat/0311066

[36] A. L. Fetter, B. Jackson, and S. Stringari, cond-mat/0407119

[37] V. Bretin, S. Stock, Y. Seurin, and J. Dalibard, Phys. Rev. Lett. 92, 050403 (2004); S. Stock, V. Bretin, F. Chevy and J. Dalibard, Europhys. Lett. 65, 594 (2004).

[38] A. Aftalion and X. Blanc, in preparation. 